\title{
Time-intensity superposition for photoinitiated polymerization of fluorinated and hyperbranched acrylate nanocomposites
}

\author{
Sara Dalle Vacche, Valérie Geiser, Yves Leterrier*, Jan-Anders E. Månson \\ Laboratoire de Technologie des Composites et Polymères (LTC), Ecole Polytechnique Fédérale de Lausanne (EPFL), CH-1015 Lausanne, Switzerland
}

\section{A R T I C L E I N F O}

\section{Article history:}

Received 2 July 2009

Received in revised form

25 November 2009

Accepted 29 November 2009

Available online 16 December 2009

\section{Keywords:}

Photopolymerization

Master curves (time-intensity

superposition)

Kinetics

\begin{abstract}
A B S T R A C T
The validity of the time-intensity superposition principle for the photoinitiated polymerization of nanocomposites based on a monofunctional fluorinated acrylate and on a multifunctional hyperbranched polyether acrylate was investigated in this work. Master curves were obtained for the conversion as a function of time, measured by photo differential scanning calorimetry, by shifting on the time axis the curves obtained at different intensities. A power-law dependence of the shift factor on the intensity was found for all materials, with exponents equal to $0.45 \pm 0.03$ for the fluorinated acrylates and to $0.71 \pm 0.05$ for the hyperbranched polyether acrylates. Consequently it is inferred that the radiant exposure reciprocity law, implying linear dependence of the shift factor on intensity, does not apply to the studied compositions. The kinetics of the photopolymerization of materials based on the fluorinated acrylate was analyzed with the autocatalytic model. The final conversion was independent on intensity and filler content. The rate constants showed for all materials a power-law dependency on intensity, with exponents similar to those of the corresponding shift factors.
\end{abstract}

(c) 2009 Elsevier Ltd. All rights reserved.

\section{Introduction}

The photoinitiated polymerization of acrylates under UV light has attracted high interest for several commercial applications, as coatings for several types of materials (including metals, wood, plastics, optical fibers, etc), adhesives and restorative dentistry formulations [1-8]. Their main advantages are solvent free formulation, low energy consumption, and ambient temperature operation. Acrylates are highly reactive and the transformation from a liquid resin to a solid polymer in the areas exposed to light is extremely fast. This characteristic makes them very good materials for photolithography for high definition images [9,10].

The nature of the acrylated monomer influences the properties of the final material, and the wide choice of acrylated monomers allows tailoring of the materials. Fluorinated chains impart water and oil repellency and therefore self-cleaning properties [11]. Hyperbranched monomers provide lower shrinkage than conventional acrylates allowing obtaining microstructures with high dimensional accuracy $[9,12]$.

The addition of inorganic nanofillers to the compositions may also provide additional benefits. For example the addition of nanosized $\mathrm{SiO}_{2}$ to photocurable formulations leads to improved

\footnotetext{
* Corresponding author. Tel.: +41 21693 4848; fax: +41 216935880 .

E-mail address: yves.leterrier@epfl.ch (Y. Leterrier).
}

mechanical properties, thermal stability, scratch resistance, and reduced shrinkage upon polymerization [13-16].

The kinetics of the photoinitiated polymerization of acrylates and methacrylates has been studied by means of photo differential scanning calorimetry (photoDSC) [17-23], real time Fourier transform infrared analysis (FT-IR) [18,24-26], photorheology [27,28], and fluorescent probe $[29,30]$. The autocatalytic model has been widely employed to describe the photopolymerization kinetics $[17,20,30,31]$.

For applications requiring fast curing it would be advantageous to benefit from the very fast polymerization rates attainable with high UV intensities, while in other cases, as for dental restoration [32], curing at lower intensity leads to higher integrity of the final material. It is therefore important to understand the influence of light intensity on the maximum conversion of photocurable resins. Some studies suggest that the final conversion does not depend on the light intensity but only on the energy dose, i.e. the product of the time of the exposure and the light intensity [33,34]. On the other hand some researchers found that the final conversion and properties of the polymerized materials depended on the light intensity $[17,21,35,36]$. The contradictory results obtained brought up the debate on the applicability to the photopolymerization of acrylates of the radiant exposure reciprocity law, stating that it is only the energy dose that determines the final effect on the light sensitive material [24]. The concept that a certain property of the polymer, namely the conversion, remains unchanged upon 
changing the intensity if the timescale is appropriately changed as well was successfully applied to UV-initiated cationic photopolymerization of an epoxy resin [37]. If the time-intensity superposition principle is applicable to the conversion of a monomer, it is possible to find the law that links intensity and time, and therefore check the validity of the reciprocity law.

The aim of this work is to determine whether the time-intensity superposition principle and the reciprocity law are applicable to the photopolymerization of acrylates by studying the curing kinetics of two different monomers, a monofunctional fluorinated acrylate and a multifunctional hyperbranched acrylate, and of nanocomposites based on these monomers.

\section{Experimental}

\subsection{Materials and sample preparation}

The monofunctional fluorinated acrylate monomer, $1 \mathrm{H}, 1 \mathrm{H}$ perfluoro-n-octyl acrylate (97\%), shown in Fig. 1a, was obtained from Exfluor Research Corporation, and contained 50 ppm methyl hydroquinone as inhibitor. AUV curable fluorosurfactant, PolyFox ${ }^{\circledR}$ PF-3320 (Fig. 1b), obtained from Polysciences, Inc. and a photoinitiator, 2,2dimethoxy-2-phenylacetophenone (99\%) (Fig. 1c), supplied by Aldrich, were dissolved into the monomer upon stirring at $50{ }^{\circ} \mathrm{C}$ for $1 \mathrm{~h}$. The concentrations were $14 \mathrm{wt} \%$ and $0.5 \mathrm{wt} \%$ respectively. The mixture prepared in this way was left to stand overnight prior to further use and will be referred to as PFOA precursor.

Cab-o-sil ${ }^{\circledR}$ M-5 untreated fumed silica (Aldrich) was used as nanofiller. It is an amorphous, colloidal silicon dioxide with a surface area of $200 \mathrm{~m}^{2} / \mathrm{g}$. The silica particles form chain-like aggregates with an average length of $0.2-0.3 \mu \mathrm{m}$. Compositions containing $1.5 \mathrm{wt} \%$ and $3 \mathrm{wt} \% \mathrm{SiO}_{2}$ were prepared (nc-PFOA). First 1.5 wt\% Cab-o-sil was thoroughly syringe mixed into the PFOA precursor. The nanocomposite mixture was then sonicated in an ultrasonic disruptor (Digital Sonifier 450, Branson) for $1.5 \mathrm{~min}$ at $20 \%$ amplitude to disagglomerate the $\mathrm{SiO}_{2}$. Compositions containing 3 wt\% nanofiller were prepared further syringe mixing the corresponding amount of Cab-o-sil into the $1.5 \mathrm{wt} \%$ mixture. The $3 \mathrm{wt} \%$ composition was too viscous to be sonicated effectively and silica aggregates are visible in the final material (Fig. 2a).

The polyfunctional monomer was based on a 3rd generation hyperbranched polyether polyol, giving a 29-functional
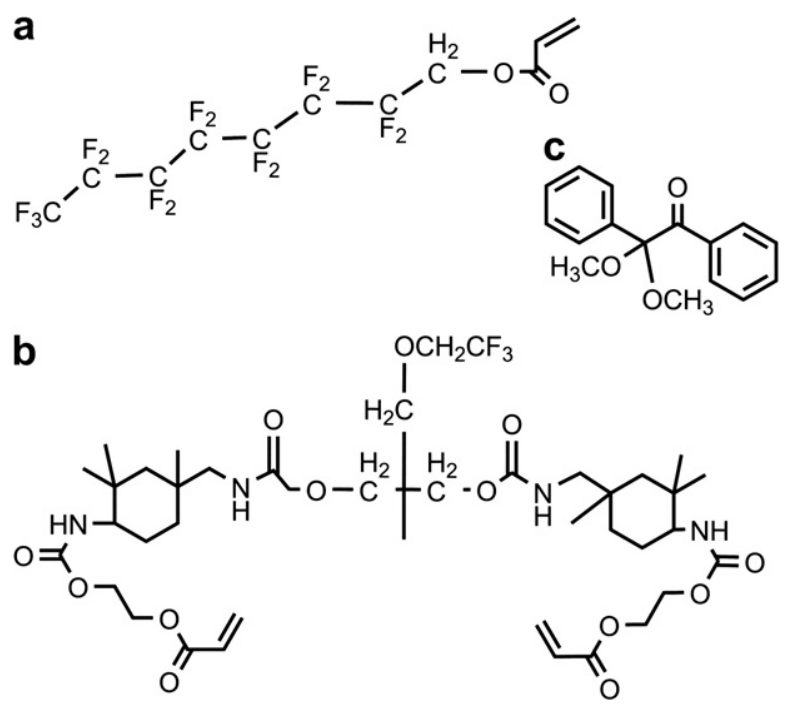

Fig. 1. Structures of (a) perfluoro-n-octyl acrylate, (b) PolyFox ${ }^{\circledR}$ P-3220 and (c) 2,2dimethoxy-2-phenylacetophenone. hyperbranched polyether acrylate (HBP, Perstorp AB). Details of the structure and physical properties of the acrylated HBP can be found elsewhere [16]. The photoinitiator was 1-hydroxy-cyclohexylphenyl-ketone (Irgacure ${ }^{\circledR} 184$, Ciba Specialty Chemicals). It was dissolved in the HBP under stirring at $70{ }^{\circ} \mathrm{C}$ for $30 \mathrm{~min}$ at a concentration equal to $1 \mathrm{wt} \%$ and showed good solubility in the acrylate monomer. Two types of amorphous silica nanofillers were used. The first, Highlink ${ }^{\circledR}$ NanO G502 (Clariant), is a suspension of $30 \mathrm{wt} \%$ monodispersed $\mathrm{SiO}_{2}$ in isopropanol. The average particle size according to the supplier is $13 \mathrm{~nm}$, which corresponds to a specific surface area of about $230 \mathrm{~m}^{2} / \mathrm{g}$. X-ray disc centrifuge (BI$\mathrm{XDC}$, Brookhaven) measurements revealed an average particle size of $23 \mathrm{~nm}$ with a standard deviation of $16 \mathrm{~nm}$ [16]. The second, Aerosil ${ }^{\circledR} \mathrm{R} 7200$ (Degussa), is a $\mathrm{SiO}_{2}$ powder with a specific surface area of about $150 \mathrm{~m}^{2} / \mathrm{g}$. The primary particle size is $12 \mathrm{~nm}$, but agglomerates up to several micrometers were observed [16]. The Aerosil particles underwent a surface treatment with methacrylsilane before use in order to promote interphase properties. Compositions with $5 \mathrm{vol} \%$ (8.7 wt\%) and $20 \mathrm{vol} \% \mathrm{SiO}_{2}$ (31 wt\%) were prepared (nc-HBP). Highlink was used as received, while Aerosil was first disagglomerated in isopropanol (ratio $1: 3$ by weight) applying ultrasound for 5 min at 70\% amplitude (Digital Sonifier 450 , Branson). The corresponding amounts of the $\mathrm{SiO}_{2}$ suspensions were then mixed with the HBP and stirred for $1 \mathrm{~h}$. The solvent was evaporated at $40^{\circ} \mathrm{C}$ under vacuum.

\subsection{Methods}

The heat of the photopolymerization reactions was measured by means of a Q100 differential scanning calorimeter (DSC) by TA Instruments, modified with a photoDSC set up. A UV lamp with a $200 \mathrm{~W}$ mercury bulb (OmniCure, 2000, Exfo, Canada) was connected to the DSC cell by means of an optical fiber. The cell was sealed with a quartz window that let the UV light pass onto the open aluminum sample pans. The sample space was flushed with nitrogen. To insure equal illumination conditions throughout the sample volume, the samples were weighed to give a thickness of $500 \mu \mathrm{m}$. The measurements were carried out at intensities ranging from 0.5 to $50 \mathrm{~mW} / \mathrm{cm}^{2}$ and at ambient temperature, the increase of temperature due to the irradiation of the lamp being less than $1{ }^{\circ} \mathrm{C}$ at the end of the reaction. The reaction was considered completed when it was no longer possible to detect a change in heat flux. The total heat of reaction was then calculated according to Hoyle [38] by integration of the area under the exothermic peak. The conversion at each point in time was calculated as the ratio of the total heat of reaction to the heat of reaction evolved at the time $t$.

Fourier transform infrared (FT-IR) spectra of the precursors and polymers were recorded with a Nicolet Magna-IR 560 spectrometer equipped with a Specac Golden Gate ATR accessory. The spectra were acquired with 32 scans and a resolution of $4 \mathrm{~cm}^{-1}$. In order to acquire the spectra of the polymerized materials, the samples polymerized in the photoDSC were taken out from the DSC pans and measured on both the top and bottom surfaces, to check for conversion gradients.

The viscosity of the $3 \mathrm{wt} \%$ nc-PFOA precursor was measured with a strain controlled rotational rheometer (ARES, Rheometric Scientific, 2kFRT transducer), with a $25 \mathrm{~mm}$ parallel plate set up at ambient temperature. The viscosity at $1 \mathrm{~Hz}$ and $1 \%$ strain was $95 \mathrm{~Pa}$ s. The viscosity of the HBP + Aerosil composites was somewhat lower (50 Pa s at 20 vol\%), while the HBP + Highlink composites showed a much higher viscosity at high filler content $\left(9 \times 10^{5}\right.$ Pa s at 20 vol\%) [16].

The thermal properties of the PFOA based precursors and polymers were analyzed by differential scanning calorimetry. The measurements were carried out between $-100{ }^{\circ} \mathrm{C}$ and $70{ }^{\circ} \mathrm{C}$ at 

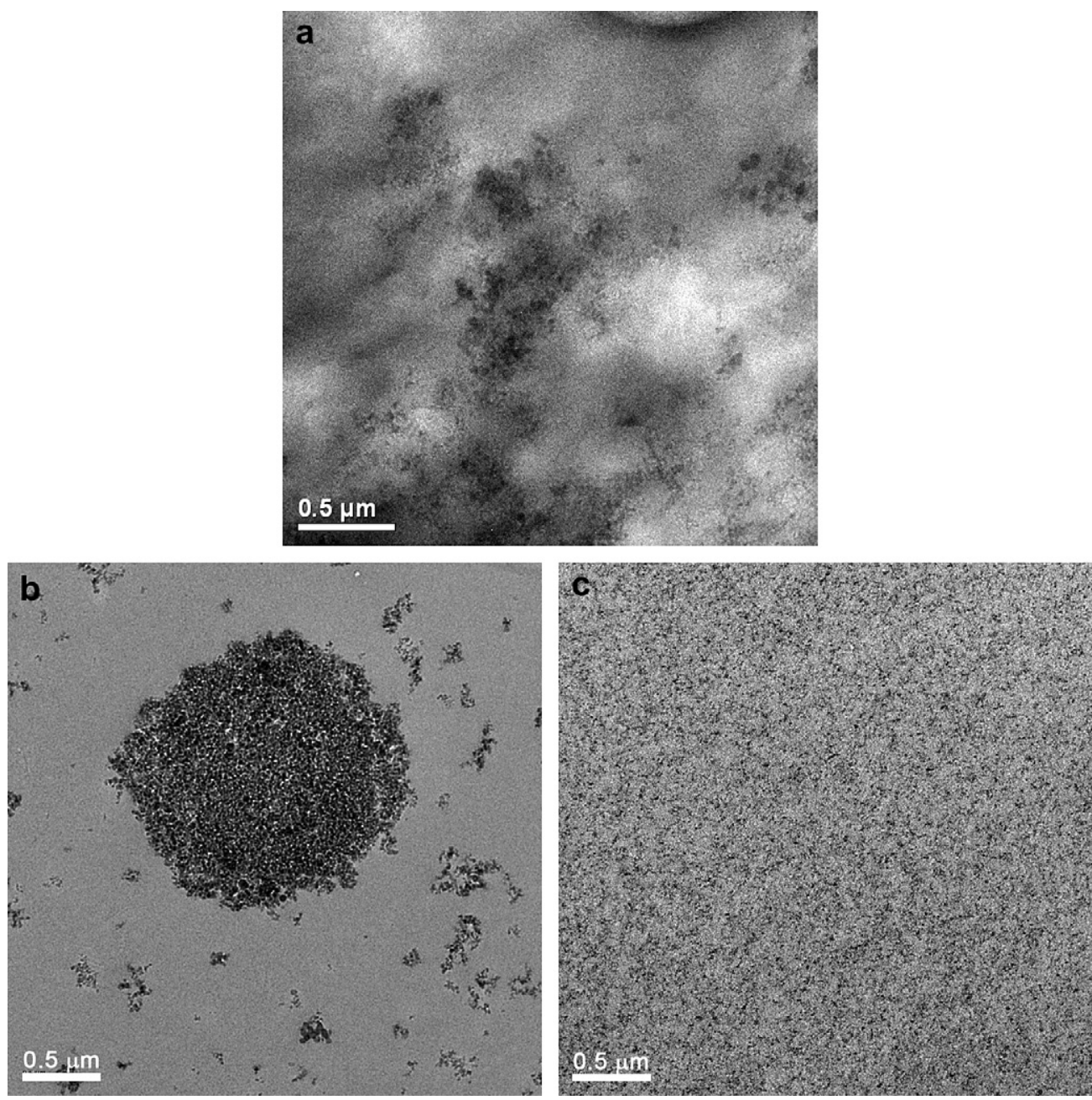

Fig. 2. TEM micrographs of (a) PFOA +3 wt $\%$ Cab-o-sil, (b) HBP +5 vol\% Aerosil and (c) HBP +5 vol\% Highlink.

a heating/cooling rate of $10{ }^{\circ} \mathrm{C} / \mathrm{min}$ (Q100 DSC, TA Instruments). Both the pure and nanocomposite PFOA precursors showed a crystallization temperature of $-43^{\circ} \mathrm{C}$. PFOA and nc-PFOA polymers, cured at $0.5 \mathrm{~mW} / \mathrm{cm}^{2}$ and $50 \mathrm{~mW} / \mathrm{cm}^{2}$ UV intensity, showed a broad glass transition around $-35{ }^{\circ} \mathrm{C}$ (middle point of the transition, $\left.T_{\mathrm{g}}\right)$, a crystallization temperature $\left(T_{\mathrm{C}}\right)$ of $29^{\circ} \mathrm{C}$ and a melting temperature $\left(T_{\mathrm{m}}\right)$ of $52^{\circ} \mathrm{C}$. The $T_{\mathrm{g}}$ of the HBP and nc-HBP polymers measured by DSC was $9{ }^{\circ} \mathrm{C}[16]$.

Dynamic Mechanical Analysis (Q800 DMA, TA Instruments) of nc-PFOA polymers containing $3 \mathrm{wt} \%$ Cab-o-sil was performed between $-50{ }^{\circ} \mathrm{C}$ and $60{ }^{\circ} \mathrm{C}$ at a heating rate of $5{ }^{\circ} \mathrm{C} / \mathrm{min}$. The excitation frequency was $1 \mathrm{~Hz}$ and the strain was $0.1 \%$. Samples $6 \mathrm{~mm}$ wide and $0.3 \mathrm{~mm}$ thick were obtained by photopolymerization under nitrogen at 5 and $40 \mathrm{~mW} / \mathrm{cm}^{2}$ for $300 \mathrm{~s}$ with the same OmniCure 2000 UV lamp used for the photoDSC experiments. Intensities were checked with a Solatell SolaCheck ${ }^{\circledR}$ UV spectrometer. For all samples the $\tan \delta$ curve showed two peaks, a first smaller broad peak at $-18{ }^{\circ} \mathrm{C}\left(T_{\mathrm{g}}\right)$ and a second higher sharp peak at $45^{\circ} \mathrm{C}$ $\left(T_{\mathrm{m}}\right)$. These results are in agreement with those obtained by DSC.

\section{Results and discussion}

\subsection{Photopolymerization}

The materials polymerized in the photoDSC apparatus or under the UV lamp were analyzed by FT-IR to check the presence of any residual acrylate double bonds. The monomers were as well analyzed for reference. Fig. 3 shows the spectrum of the PFOA + 3 wt\% Cab-osil precursor and of the same material polymerized at $2.5 \mathrm{~mW} / \mathrm{cm}^{2}$ intensity (top and bottom surfaces). Fig. 4 shows the spectra of the HBP precursor and of the same material photopolymerized at $12 \mathrm{~mW} / \mathrm{cm}^{2}$ intensity. The doublet peak near 1635 and $1620 \mathrm{~cm}^{-1}$ corresponding to $\mathrm{C}=\mathrm{C}$ in acrylates [39] was used to check conversion [40]. The peaks at $1750 \mathrm{~cm}^{-1}$ for PFOA based materials and at $1725 \mathrm{~cm}^{-1}$ for $\mathrm{HBP}$ were assigned to $\mathrm{C}=\mathrm{O}$ stretching [39].

For all the PFOA and nc-PFOA polymers the FT-IR spectra did not show the doublet peak at 1620 and $1635 \mathrm{~cm}^{-1}$, indicating that the final concentration of not converted acrylate double bond was below detection limit. The conversion may then be considered complete. For the HBP polymers the doublet peak was still visible indicating a lower degree of conversion.

The spectra taken on the top and bottom surface of the polymerized samples are similar for HBP, while for PFOA and nc-PFOA they show some remarkable differences. The bottom surface spectrum is similar to the monomer spectrum, except for the disappearance of the $\mathrm{C}=\mathrm{C}$ peaks and increase of the $\mathrm{C}-\mathrm{C}$ peaks. The top surface spectra however show a new peak at $1725 \mathrm{~cm}^{-1}$ and a decrease of the peak at $1750 \mathrm{~cm}^{-1}$. The shift of the $\mathrm{C}=0$ peak from 1750 to $1725 \mathrm{~cm}^{-1}$ has been attributed to the formation of hydrogen bonds between $\mathrm{C}=\mathrm{O}$ and $\mathrm{OH}$ groups [41]. In the upper side spectra also a broad band at $3500-3200 \mathrm{~cm}^{-1}$ appears, attributed to $\mathrm{OH} \cdots \mathrm{O}$ stretching [39]. 
PFOA $+3 \%$ Cab-o-sil

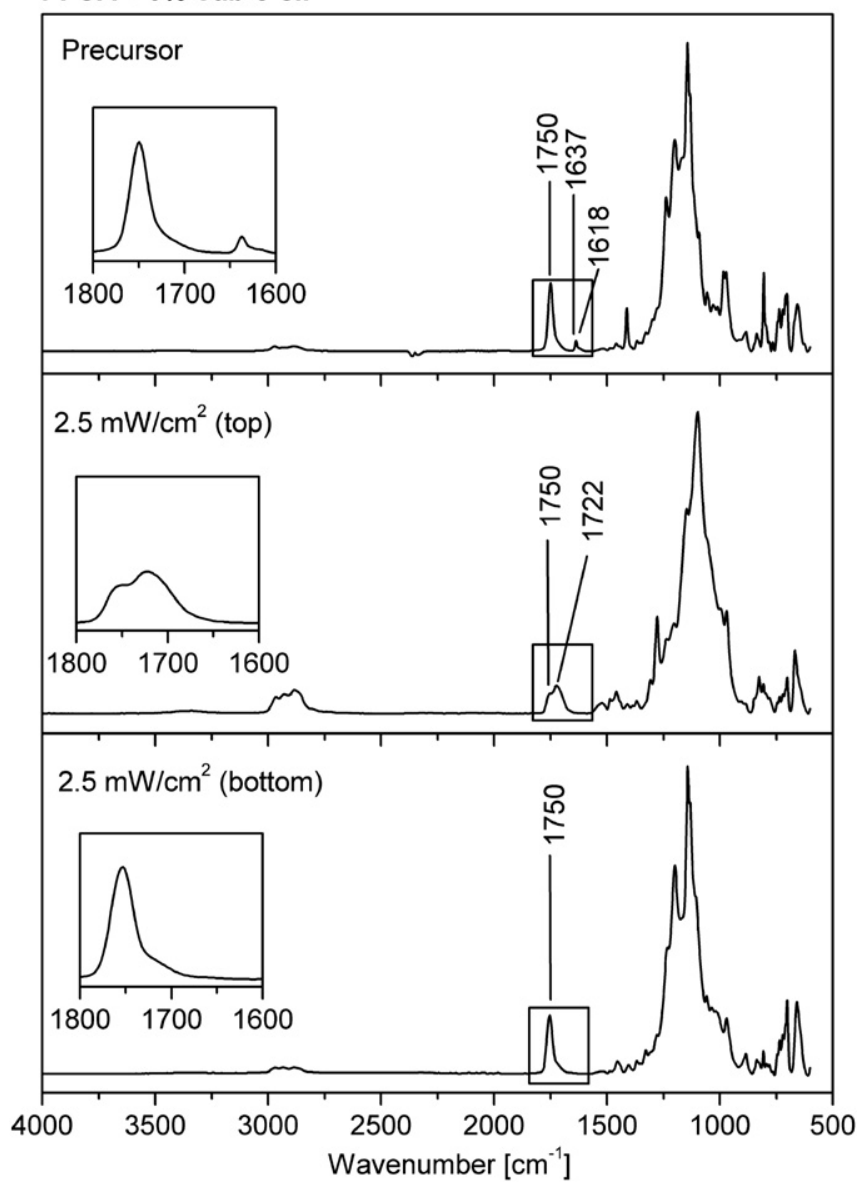

Fig. 3. FT-IR spectra of PFOA +3 wt\% Cab-o-sil, precursor and photopolymerized at $2.5 \mathrm{~mW} / \mathrm{cm}^{2}$ (top and bottom surfaces).

The heat flow recorded by the photoDSC was used to follow the advancement of the photopolymerization reaction and calculate conversion. As the FT-IR spectra of the photopolymerized HBP based materials indicated the presence of not converted acrylate double bonds, the double bond conversion at each point in time was calculated as the ratio of the measured heat of reaction to the theoretical total heat of reaction [16]. The latter was calculated as the number of acrylate double bonds per gram of formulation times the heat evolved per converted double bond, i.e. $86.2 \mathrm{~kJ} / \mathrm{mol}$ [42].

The maximum heat of reaction obtained by photocalorimetry for the PFOA based systems was higher than the theoretical heat calculated as described above. The moles of acrylate per gram of formulation were calculated from the molecular weights and acrylate numbers given by the suppliers. The presence of lower molecular weight or higher functionality impurities may partly account for the difference between the measured and theoretical heat. Some uncertainty in the determination of the theoretical heat per double bond, that was measured for the thermal polymerization of lauryl acrylate [42], must also be considered. As FT-IR showed no residual acrylate double bonds in the photopolymerized PFOA based materials, the maximum measured heat of reaction obtained for each of these systems was considered to correspond to complete conversion. The conversion during the photopolymerization was then calculated at each moment as the ratio of the heat of reaction to the maximum heat.

The acrylate double bond conversion as a function of time at different UV intensities for the PFOA and nc-PFOA with $1.5 \mathrm{wt} \%$ and

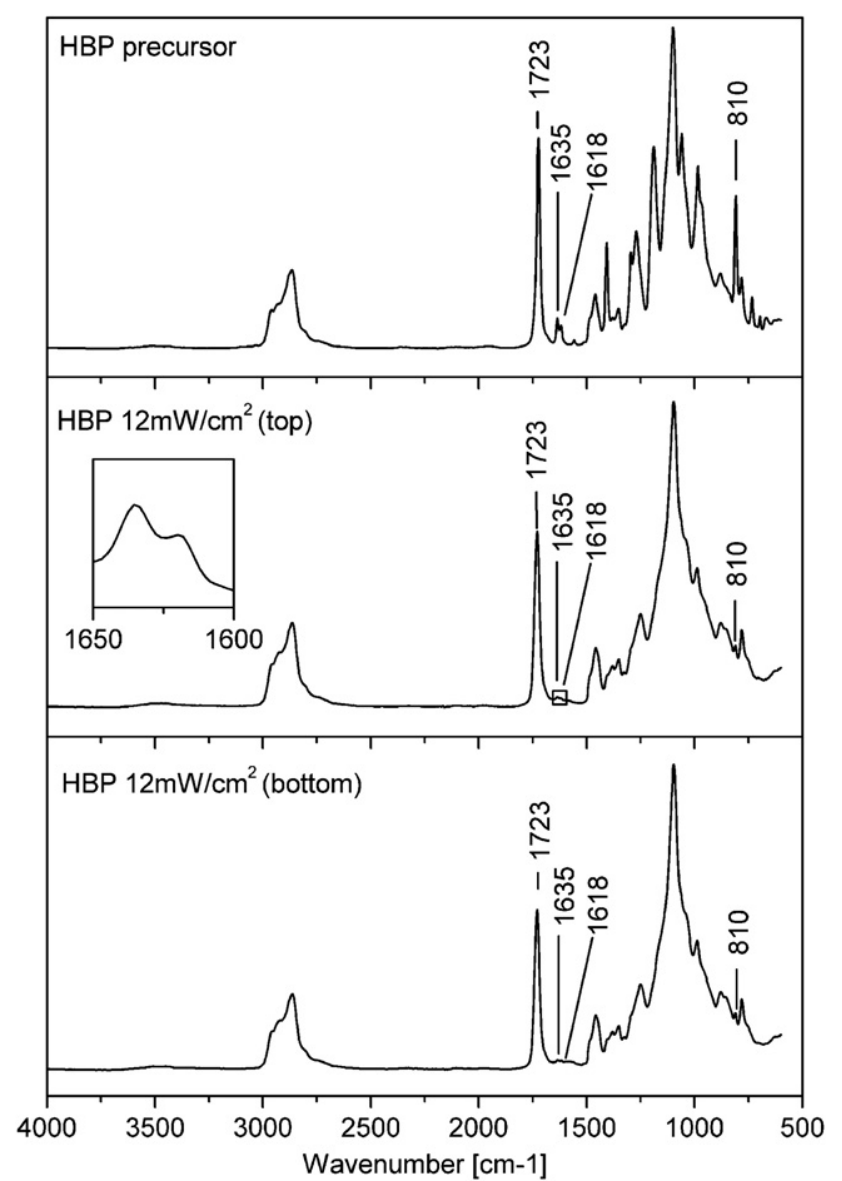

Fig. 4. FT-IR spectra of HBP, precursor and photopolymerized at $12 \mathrm{~mW} / \mathrm{cm}^{2}$ intensity.

3 wt\% Cab-o-sil are reported in Fig. 5a,c,e and for HBP +5 vol\% Highlink in Fig. 6a. Similar curves for HPB and HBP +20 vol\% Highlink and Aerosil nanocomposites are reported in a previous paper [16]. The maximum conversion for PFOA based materials resulted in all cases higher than 0.9 while for the HBP based materials was lower (between 0.65 and 0.73 [16]) as expected for multifunctional monomers.

After an induction time, which was shorter at the higher intensities ( $10 \mathrm{~s}$ at $0.5 \mathrm{~mW} / \mathrm{cm}^{2}$, less than $1 \mathrm{~s}$ at $50 \mathrm{~mW} / \mathrm{cm}^{2}$ ) and may be attributed to the formation of initiator-derived radicals and in the case of HBP also to the inhibiting action of dissolved oxygen, the reaction rate rapidly increased. This is the so-called autoacceleration or gelation effect [35]: due to the increased viscosity the mobility of the long-chain radical species is reduced, therefore reducing the termination rate as the recombination of two radical chains is not favored, while the small initiator radicals and the monomers can still move freely and continue the polymerization.

The reaction rate reaches a maximum value $\left(\alpha_{\max }^{\prime}\right)$ at a conversion of $0.2-0.3$ (Fig. 7a) then it decreases again (autodeceleration) while the reaction goes on until the maximum conversion.

A third stage in which polymerization continues at very low rate, attributed to vitrification [35] was not present, in agreement with the fact that the $T_{\mathrm{g}}$ of the polymers used in this study is lower than the polymerization temperature. However for PFOA based materials the reaction rate versus conversion curve shows a shoulder at a conversion of about 0.8 (Fig. 9). A full explanation for this has not yet been found. A first hypothesis is that some crystallization took place, as the crystallization temperature of the 

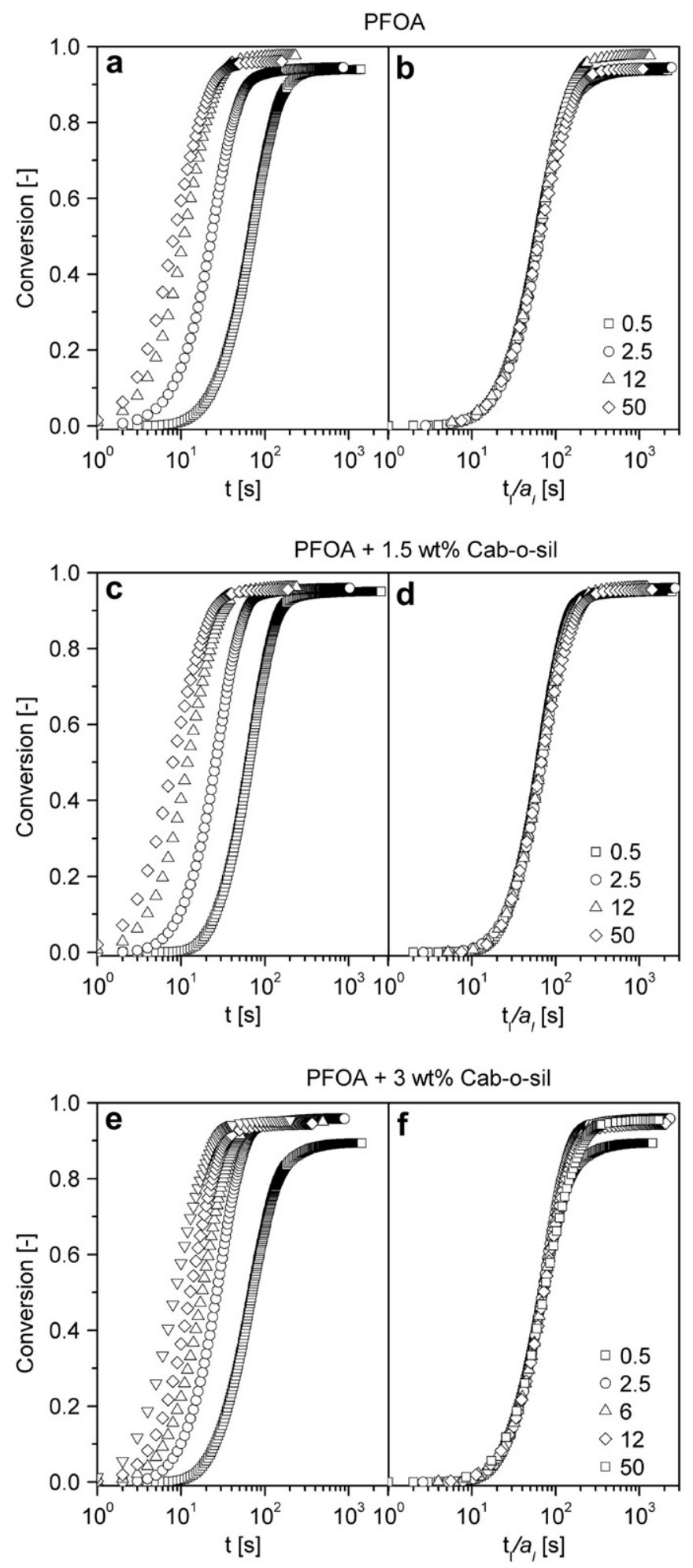

Fig. 5. (a, c, e) Conversion versus time and (b, d, f) time-intensity superposition for PFOA and nc-PFOA materials. (Intensities from 0.5 to $50 \mathrm{~mW} / \mathrm{cm}^{2}$ ).

PFOA based polymers is close to the polymerization temperature. However the crystallization heat, as measured by DSC on the polymerized samples, did not exceed $7 \%$ of the total heat evolved. A second hypothesis is that a side reaction started, possibly at the upper surface of the sample, which may explain the shift of the $\mathrm{C}=\mathrm{O}$ peak. Both hypotheses may contribute to explain the high total heat of reaction.

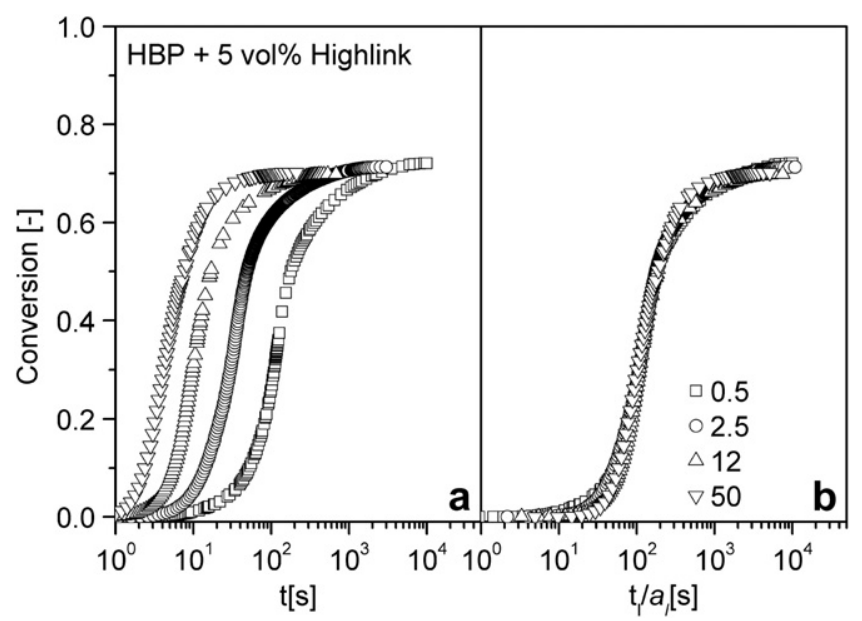

Fig. 6. (a) Conversion versus time and (b) time-intensity superposition of $\mathrm{HBP}+5 \mathrm{vol} \%$ Highlink. (Intensities from 0.5 to $50 \mathrm{~mW} / \mathrm{cm}^{2}$ ).

The reaction rate was, as expected, higher at the higher intensities. However, the UV intensity did not influence the final conversion of the PFOA based materials (Fig. 7b). The same was found by Geiser et al. [16] for the HBP based materials both with the Aerosil and the Highlink nanofillers.

Also the nanofiller content was found not to influence the final conversion of the PFOA based materials. The same result was obtained for the HBP + Aerosil nanocomposites, while HBP + Highlink nanocomposites showed a conversion decreasing with the nanofiller volume fraction [16]. The different behaviour of the Aerosil and Highlink composites was explained in terms of the different degree of dispersion of the nanosilica. In HBP + Aerosil nanocomposites the filler distribution was inhomogeneous, showing aggregates, while in the HBP + Highlink it was homogeneous (Fig. 2b,c). The better dispersion of the nanofiller highly increased the viscosity of the HBP + Highlink nanocomposite, especially at the highest concentration. The mobility of reacting species at the beginning of the reaction was therefore reduced and

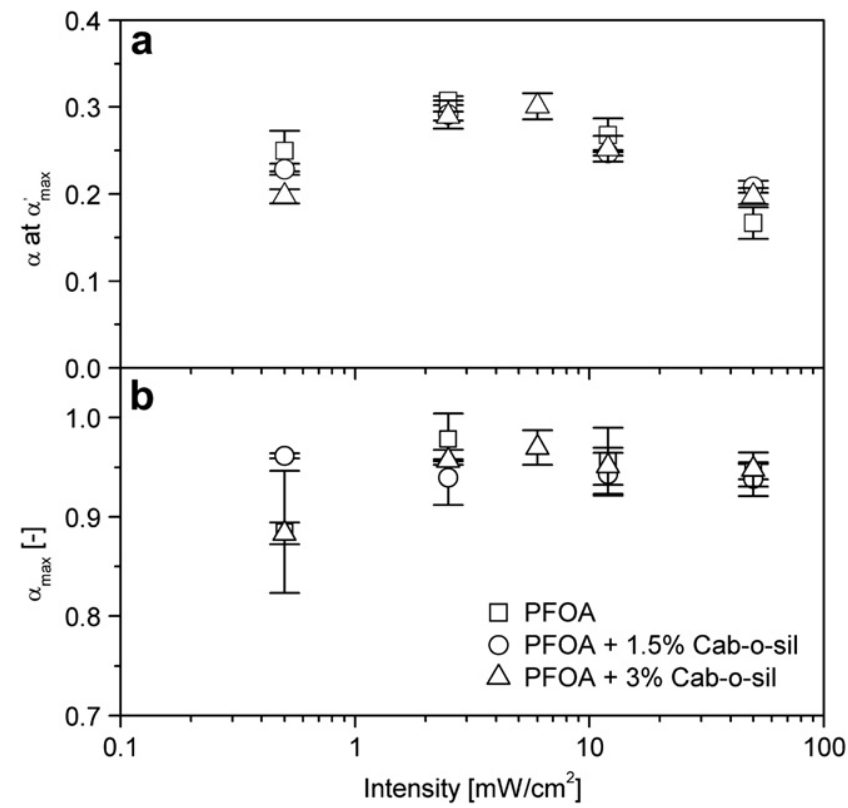

Fig. 7. (a) Conversion at the maximum conversion rate versus intensity and (b) maximum conversion versus intensity for PFOA and nc-PFOA materials. 
more radicals were trapped in the growing network. The PFOA nanocomposites showed a non homogeneous dispersion of the nanofiller and a viscosity similar to HBP + Aerosil composites, therefore supporting this hypothesis.

\subsection{Time-intensity superposition}

The time-intensity superposition principle may be written as:

$\alpha\left(t_{1}, I_{1}\right)=\alpha\left(t_{0}, I_{0}\right) \Leftrightarrow t_{1}=\frac{t_{0}}{a_{I}}$

where $\alpha$ is the conversion, $t$ the time, I the irradiation intensity and $a_{I}$ the time-intensity shift factor. The validity of this approach was demonstrated by Corcione et al. [37] for the photopolymerization of non-vitrifying epoxy based resin.

For each PFOA and HBP composition a single master curve was obtained by a horizontal shift of the conversion versus time curves at different intensities according to equation (1). The shift factors were determined using the data at $0.5 \mathrm{~mW} / \mathrm{cm}^{2}$ as reference, therefore the shift factor at $0.5 \mathrm{~mW} / \mathrm{cm}^{2}$ is unity. The shifted curves are shown for PFOA and nc-PFOA in Fig. 5b,d,f and for HBP + 5 vol\% Highlink in Fig. 6b.

For the PFOA based materials the time-intensity superposition is excellent until a conversion of about 0.7 , and then the shifted curves start diverging. A possible reason for this may be the onset of crystallization. For HBP based materials the time-intensity superposition principle holds very well between 0.1 and 0.5 conversion. The discrepancy in the initial part of the curves may be explained with a delay in the measurement of the evolved heat by the DSC which is emphasized at the high intensities when the heat flux is higher.

Both for PFOA and HBP based materials the filler content does not affect the shift factors that show a power-law dependence on the intensity (Fig. 8):

$a_{I}=a_{0} I^{b}$

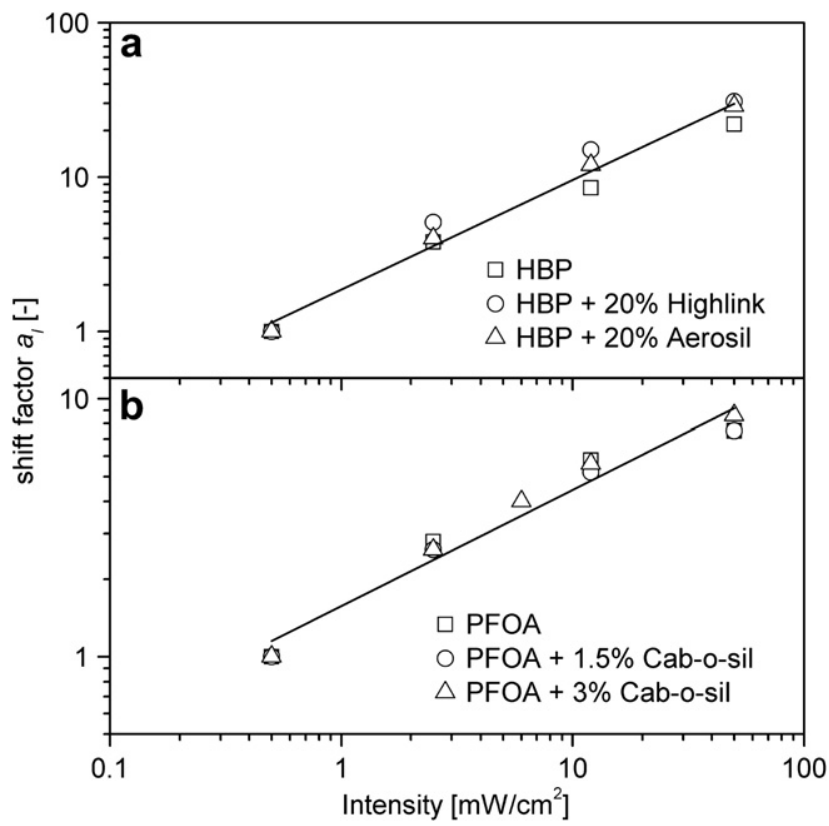

Fig. 8. Time-intensity-shift factors for (a) HBP and composites containing $20 \mathrm{vol} \%$ Highlink and Aerosil and (b) PFOA and nc-PFOA. The power-law fits are represented by the continuous lines.
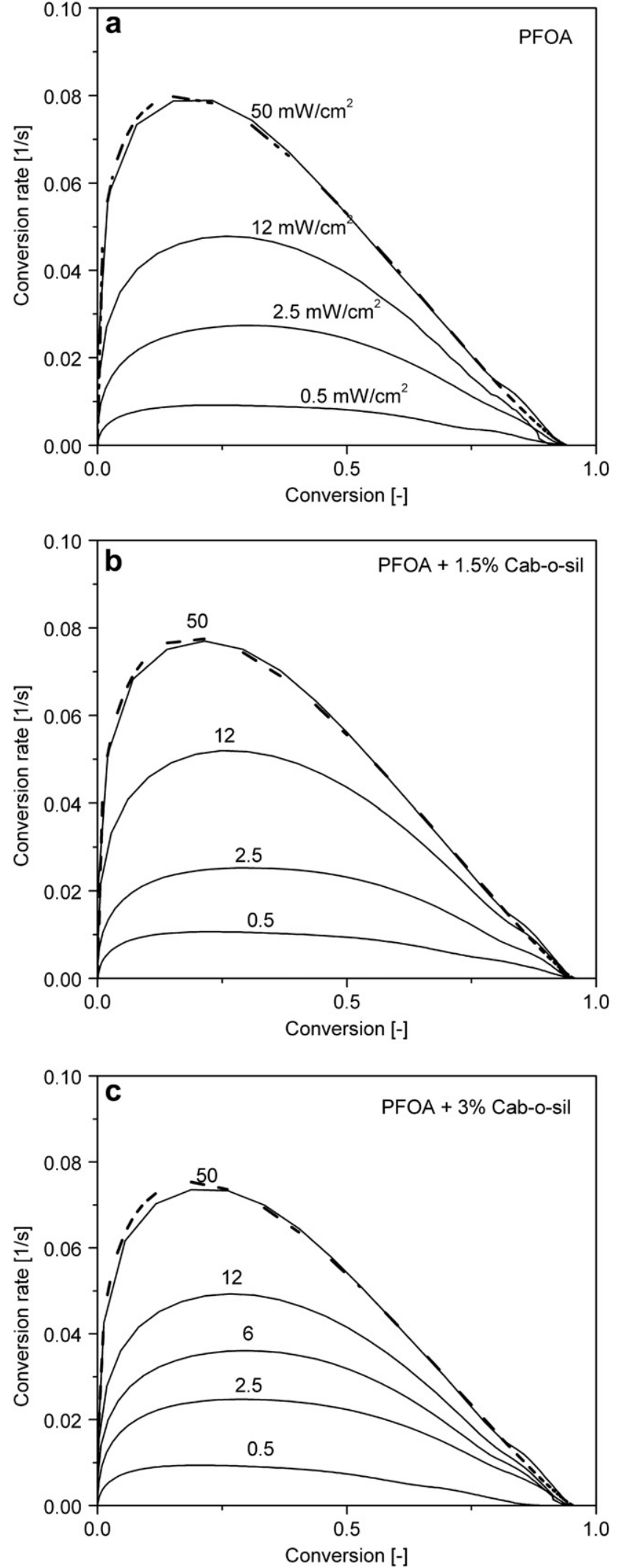

Fig. 9. Conversion rate versus conversion for (a) PFOA, (b) PFOA +1.5 wt $\%$ Cab-o-sil and (c) PFOA $+3 \mathrm{wt} \%$ Cab-o-sil at different intensities (continuous lines) and modelling of conversion rate versus conversion (dashed lines) at $50 \mathrm{~mW} / \mathrm{cm}^{2}$. 
For HBP and the composites containing Highlink and Aerosil the exponent $b$ is equal to $0.71 \pm 0.05$ and for PFOA and nc-PFOA it is equal to $0.45 \pm 0.03$.

\subsection{Kinetics}

In order to asses if this result is consistent with the kinetics of photopolymerization of the investigated materials a kinetic model that correctly describes the reaction is needed. The autocatalytic model has been successfully applied to the photopolymerization kinetics of acrylates. This is a phenomenological model based on the work of Kamal [43] for thermal cure of polyesters. Although acrylate photopolymerization is not autocatalytic but autoaccelerated, the shape of the curve of reaction rate versus conversion is the same.

The autocatalytic model can be expressed as:

$\frac{\mathrm{d} \alpha}{\mathrm{d} t}=k \alpha_{r}^{m}\left(1-\alpha_{r}\right)^{n}$

with

$\alpha_{r}=\frac{\alpha}{\alpha_{\max }}$

where $\alpha$ is the conversion at any time, $\alpha_{\max }$ is the maximum conversion, $k$ is the rate constant, $m$ is the autocatalytic exponent and $n$ the reaction order. The autocatalytic exponent takes into account the autoacceleration of the photopolymerization reaction caused by the immobilization of the polymer chains in the forming network due to an increase in viscosity that prevents recombination of the polymer radicals, decreasing the termination rate.

The experimental curves for the conversion rate as a function of conversion for the PFOA based materials at different intensities are reported in Fig. 9. In the same figures are reported as example the curves obtained with the autocatalytic model for an intensity of $50 \mathrm{~mW} / \mathrm{cm}^{2}$.

As the reason for the shoulder at about 0.8 conversion is not clear, the fitting of the experimental data to obtain the values of the parameters of the autocatalytic model was done taking in to account only the part of the experimental curves before the shoulder.

For all the PFOA based systems the reaction order $n$ was found to be independent of the intensity within the experimental scatter, with a value close to 1 , while the autoacceleration exponent $m$ was found to have a value close to 0.4 and very slightly decrease with intensity (Fig. 10a). This may be due to a slightly reduced viscosity effect at higher intensities. The total reaction order $(m+n)$ was between 1.4 and 1.6. Similar results were obtained by Khudyakov and co-workers [30], who found for photopolymerization of monofunctional acrylates in $\mathrm{N}_{2}$ atmosphere values of $n$ between 1.2 and 1.4 and of $m$ between 0.4 and 0.55 .

The values of the rate constant $k$ at the different intensities are plotted in Fig. 10b. The dependence of the rate constant on the UV intensity can be expressed as

$k=k_{0} \cdot I^{\beta}$

where $I$ is the UV intensity. The value of the exponent $\beta$ is linked to the termination mechanism [44]. When $\beta<0.5$ primary radical termination, i.e. reaction of an initiator radical with a polymer radical, is the predominant mechanism. $\beta=0.5$ indicates second order termination, i.e. reaction of two polymer radicals, while $\beta=1$ indicates first order termination, i.e. trapping of the radical end in the forming network or recombination with oxygen. For $0.5<\beta<1$ first order and second order termination happen in parallel.

For the PFOA materials the constant $k_{0}$ and the exponent $\beta$ were found for the range of compositions studied to be independent of the filler content. The value of the exponent $\beta$ was found to be 0.42 ,

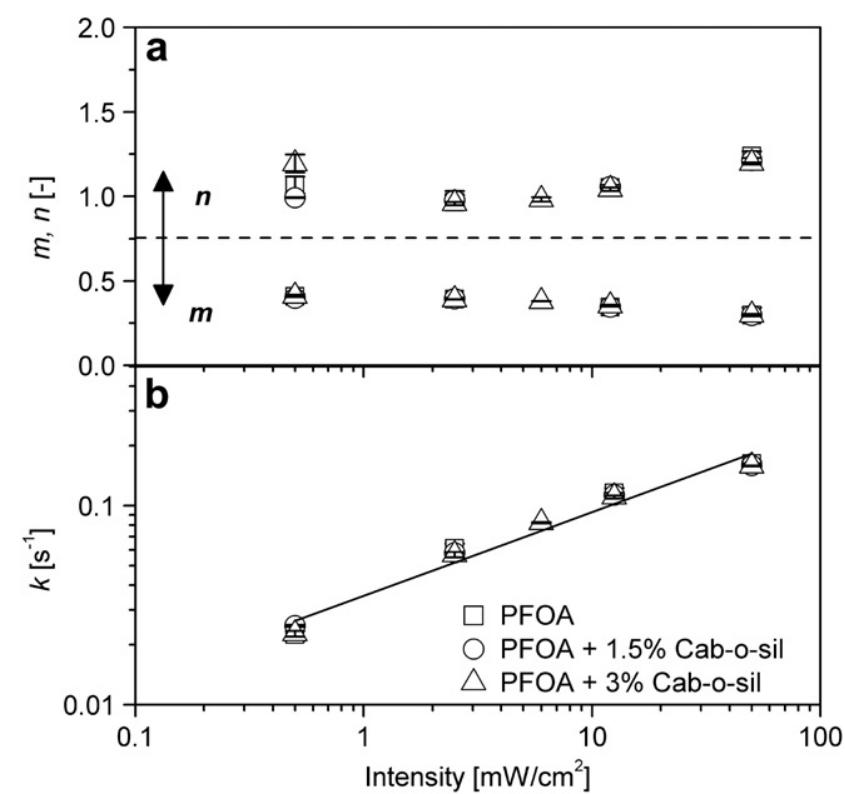

Fig. 10. (a) Autocatalytic exponent, $m$, reaction order, $n$, and (b) rate constant, $k$, and power-law fit of $k$ (continuous line) for PFOA based systems.

indicating primary radical termination. This result is in agreement with those of Schmidt et al. [17] and Timpe et al. [45] who also found $\beta<0.5$.

The modelling of HBP polymers has been published previously by Geiser et al. [16]. The value of the rate constant $k_{0}$ was found to be only slightly dependent on filler content, but strongly dependent on intensity and the exponent $\beta$ was between 0.5 and 1 . The higher values found were attributed to higher amounts of dissolved oxygen and to the higher number of trapped radicals in the hyperbranched structure compared to the monofunctional acrylate.

\subsection{Discussion}

It is evident that the values obtained for the exponent $b$ of the shift factor are equal to the values of the exponent $\beta$ of the rate constants. In fact assuming that the kinetic law chosen to model the photopolymerization (equation (1)) is correct one can write, at constant temperature:

$\frac{\mathrm{d} \alpha}{\alpha_{r}^{m}\left(1-\alpha_{r}\right)^{n}}=k(I) \mathrm{d} t$

Integration of this equation leads to:

$\int \frac{\mathrm{d} \alpha}{\alpha_{r}^{m}\left(1-\alpha_{r}\right)^{n}}=\int k(I) \mathrm{d} t=k(I) \cdot t$

If $\alpha\left(t_{0}, I_{0}\right)=\alpha\left(t_{1}, I_{1}\right)$ then as the left side of equation (7) only depends on $\alpha$ it follows that:

$k\left(I_{0}\right) \cdot t_{0}=k\left(I_{1}\right) \cdot t_{1}$

Substituting the power-law dependence of $k$ (equation (3)) in equation (8) one finds that:

$k_{0} \cdot I_{0}^{\beta} \cdot t_{0}=k_{0} \cdot I_{1}^{\beta} \cdot t_{1} \Rightarrow t_{1}=I_{0}^{\beta} \cdot \frac{t_{0}}{I_{1}^{\beta}}$

Comparing this equation to equation (2) one can easily see that $b$ must be equal to $\beta$, as it has been found for PFOA and HBP photopolymerizations. 
This result also points out that the radiation dose equivalence principle that can be expressed as:

Property $=f(t \cdot I)$

is not applicable in this case. In fact to have the same conversion it is the product $\left(t \cdot I^{\beta}\right)$ that must be constant. It therefore descends that those systems for which bimolecular or primary radical termination $(\beta<<1)$ are predominant may not abide by the radiation dose equivalence law. As monomolecular termination $(\beta=1)$ is favoured by the restriction of the movement of the macroradicals, due to high viscosity and dense network formation, small monomers with low functionality and low viscosity are likely to highly deviate from the radiation dose equivalence principle. A further confirmation is given by the curing behavior, before vitrification, of DPHA (dipentaerythritol penta/hexaacrylate), a monomer with low viscosity and fairly low functionality for which Schmidt et al. found predominant primary radical and bimolecular termination with $\beta=0.35$ [17], and for which we found that the shift factor $b$ was $\sim 0.32$. These results are in agreement with the findings of Feng and Suh [24], who found that the power dose equivalence was respected in the case of monomolecular termination $(\beta=1)$, while for bimolecular termination $(\beta=0.5)$ it was not.

\section{Conclusions}

The time-intensity superposition principle was applied to the photopolymerization of the fluorinated acrylate precursors and of hyperbranched acrylate monomers, without and with addition of $\mathrm{SiO}_{2}$ nanofiller. All the conversion versus time curves could be shifted to obtain a master curve. The shift coefficients were practically independent from $\mathrm{SiO}_{2}$ content. They showed power-law dependence from UV intensity with exponents equal to $0.45 \pm 0.03$ and $0.7 \pm 0.05$ for PFOA and HBP compositions respectively. These values correspond to those found for the same compositions for the exponent of the power-law dependence of the reaction rate $k$. This result shows that the power dose equivalence principle is not applicable for the photopolymerization of these compounds.

The study of the influence of UV intensity on the photopolymerization of acrylate based systems was reported in this paper. The reaction rate was higher at the higher UV intensities, however the final conversion was not influenced by the UV intensity. An autocatalytic model was used to describe the photopolymerization kinetics of the PFOA based systems. The reaction order was found to be close to 1 and independent on UV intensity and filler content. The autocatalytic exponent was close to 0.3-0.4, independent on filler content and very slightly decreasing with UV intensity. The overall reaction order was between 1.4 and 1.6. The rate constant was found to be independent on filler content and to have a power-law dependence on intensity $k=k_{0} \cdot I^{\beta}$. The exponent $\beta$ was equal to 0.42 , i.e. lower than 0.5 , indicating prevalence of primary termination mechanism.

\section{Acknowledgements}

The authors would like to thank Konarka Technologies and the Swiss National Science Foundation (SNF project \# 200020-111706) for financial support. They also acknowledge Perstorp Specialty Chemicals for the supply of materials and useful advices and the Centre Interfacultés de Microscopie Electronique (CIME) of the EPFL for technical assistance during the TEM observations.

\section{References}

[1] Dufour P. State-of-the-art and trends in the radiation-curing market. In: Fouassier J-P, Rabek JF, editors. Radiation curing in polymer science and technology, vol. 1. Barking, Essex: Elsevier Science Publishers, Ltd; 1993. p. $1-48$.

[2] Gloeckner P, Jung T, Struck S, Studer K. Radiation curing: coatings and printing inks. Hannover: Vincentz Network GmbH \& Co KG; 2008.

[3] Decker C. Macromolecular Rapid Communications 2002;23(18):1067-93.

[4] Roose P, Fallais I, Vandermiers C, Olivier MG, Poelman M. Progress in Organic Coatings 2009;64(2-3):163-70.

[5] Huang ZG, Shi WF. European Polymer Journal 2007;43(4):1302-12.

[6] Luciani A, Plummer CJG, Gensler R, Månson JAE. Journal of Coatings Technology 2000;72(909):161-3.

[7] Rayss J, Widomski J, Luzinov I, Voronov A, Minko S. Journal of Applied Polymer Science 1998;67(11):1913-23.

[8] Güngör A, Kayaman-Apohan N, Mert A, Kahraman MV. Journal of Polymer Research 2008;15(5):389-95.

[9] Schmidt LE, Yi S, Jin YH, Leterrier Y, Cho YH, Månson JAE. Journal of Micromechanics and Microengineering 2008;18(4):8.

[10] Decker C. Polymer International 1998;45(2):133-41.

[11] Li ZR, Fu KJ, Wang LJ, Liu F. Journal of Materials Processing Technology 2008; 205(1-3):243-8.

[12] Jin YH, Cho YH, Schmidt LE, Leterrier Y, Månson JAE. Journal of Micromechanics and Microengineering 2007;17(6):1147-53.

[13] Di Gianni A, Bongiovanni R, Turri S, Deflorian F, Malucelli G, Rizza G. Journal of Coatings Technology and Research 2009;6(2):177-85.

[14] Lee CK, Don TM, Lai WC, Chen CC, Lin DJ, Cheng LP. Thin Solid Films 2008; 516(23):8399-407.

[15] Bauer F, Mehnert R. Journal of Polymer Research 2005;12(6):483-91.

[16] Geiser V, Leterrier Y, Månson JAE. Journal of Applied Polymer Science 2009;114(3):1954-63.

[17] Schmidt LE, Leterrier Y, Schmah D, Månson JAE, James D, Gustavsson E, et al. Journal of Applied Polymer Science 2007;104(4):2366-76.

[18] Wu KC, Halloran JW. Journal of Materials Science 2005;40(1):71-6.

[19] Khudyakov IV, Fox WS, Purvis MB. Industrial \& Engineering Chemistry Research 2001;40(14):3092-7.

[20] Lecamp L, Youssef B, Bunel C, Lebaudy P. Polymer 1999;40(6):1403-9.

[21] Lecamp L, Youssef B, Bunel C, Lebaudy P. Polymer 1997;38(25):6089-96.

[22] Andrzejewska E, Andrzejewski M. Journal of Polymer Science, Part A: Polymer Chemistry 1998;36(4):665-73.

[23] Doornkamp AT, Vanekenstein G, Tan YY. Polymer 1992;33(13):2863-7.

[24] Feng L, Suh BI. Macromolecular Chemistry and Physics 2007;208(3):295-306.

[25] Scherzer T. Applied Spectroscopy 2002;56(11):1403-12.

[26] Scherzer T, Decker U. Real-time FTIR-ATR spectroscopy to study the kinetics of ultrafast photopolymerization reactions induced by monochromatic UV light. In: third International Symposium on Advanced Infrared and Raman Spectroscopy (AIRS III). Vienna, Austria: Elsevier Science Bv; 1998. p. 385-98.

[27] Lee JK, Choi JY, Lim BS. Polymer Journal 2003;35(10):778-84.

[28] Schmidt LE, Leterrier Y, Vesin JM, Wilhelm M, Månson JAE. Macromolecular Materials and Engineering 2005;290(11):1115-24.

[29] Serrano B, Levenfeld B, Bravo J, Baselga J. Polymer Engineering and Science 1996;36(2):175-81.

[30] Khudyakov IV, Legg JC, Purvis MB, Overton BJ. Industrial \& Engineering Chemistry Research 1999;38(9):3353-9.

[31] Lecamp L, Youssef B, Bunel C, Lebaudy P. Nuclear Instruments \& Methods in Physics Research Section B-Beam Interactions with Materials and Atoms 1999; 151(1-4):285-9.

[32] Unterbrink GL, Muessner R. Journal of Dentistry 1995;23(3):183-9.

[33] Emami N, Soderholm KJM, Berglund LA. Journal of Dentistry 2003;31(3): 189-96.

[34] Neves AD, Discacciati JAC, Orefice RL, Yoshida MI. Journal of Biomedical Materials Research Part B-Applied Biomaterials 2005;72B(2):393-400.

[35] Kloosterboer JG. Advances in Polymer Science 1988;84:1-61.

[36] Kloosterboer JG, Lijten G. Polymer 1987;28(7):1149-55.

[37] Corcione CE, Greco A, Maffezzoli A. Polymer 2005;46(19):8018-27.

[38] Hoyle CE. In: Pappas SP, editor. Radiation curing: science and technology. New York: Plenum Press; 1992. p. 57-130.

[39] Colthup NB, Daly LH, Wiberley SE. In: Introduction to infrared and Raman spectroscopy. 3rd ed. Boston: Academic Press; 1990.

[40] Factor A, Tilley MG, Codella PJ. Applied Spectroscopy 1991;45(1):135-8.

[41] Mikhaylova Y, Adam G, Haussler L, Eichhorn KJ, Voit B. Journal of. Molecular Structure 2006;788(1-3):80-8

[42] Moore JE. Polymerization of multifunctional acrylates and methacrylates. In: Labana S, editor. Chemistry and properties of crosslinked polymers. New York: Academic Press; 1977. p. 535-46.

[43] Kamal MR. Polymer Engineering and Science 1974;14(3):231-9.

[44] Andrzejewska E. Progress in Polymer Science 2001;26(4):605-65.

[45] Timpe HJ, Strehmel B, Roch FH, Fritzsche K. Acta Polymerica 1987;38(4): 238-44. 